\title{
The inactive and active forms of the pyrroloquinoline quinone-alcohol dehydrogenase of Gluconacetobacter diazotrophicus: a comparative study
}

\author{
Saúl Gómez-Manzo, ${ }^{1}$ Irene Patricia del Arenal-Mena, ${ }^{2}$ José Edgardo Escamilla ${ }^{3}$ \\ ${ }^{1}$ Laboratorio de Bioquímica-Genética, Torre de investigación, Instituto Nacional de Pediatría; \\ ${ }^{2}$ Departamento de Bioquimica, Facultad de Medicina; ${ }^{3}$ Instituto de Fisiología Celular, Universidad \\ Nacional Autónoma de México, Ciudad Universitaria, México
}

\begin{abstract}
Gluconacetobacter diazotrophicus as a member of the acetic acid bacteria group, oxidize alcohol to acetic acid through two sequential reactions catalyzed by the alcohol dehydrogenase (ADH) and the aldehyde dehydrogenase, both enzymes are membrane-bound and oriented to the periplasmic space. ADH is a quinohemoprotein carrying one pyrroloquinoline quinone moiety, one [2Fe:2S] cluster and four c-type cytochromes, as prosthetic groups. In recent years has been described the presence of the inactive $\mathrm{ADH}(\mathrm{ADHi})$ in the acetic acid bacteria. In the present review we make a comparative study of the molecular and catalytic properties of the active and inactive forms of ADH purified from $G$. diazotrophicus, variation in the redox state of enzymes as purified could explain the notorious differences seen in the activity power of the compared enzymes.
\end{abstract}

\footnotetext{
Correspondence: José Edgardo Escamilla, Instituto de Fisiología Celular, Universidad Nacional Autónoma de México, Ciudad Universitaria 04510, México, D.F. Tel. +52.555 .622 .5627 - Fax: +52.555 .622 .5630 .

E-mail: eescami@ifc.unam.mx
}

Key words: active and inactive alcohol dehydrogenase, $c$-type cytochrome, Gluconacetobacter diazotrophicus, pyrroloquinoline quinone.

Dedication: this work is dedicated to Prof. Edgardo Escamilla who started the research but unfortunately passed away. The authors developed and concluded his project which is published in his honor and memory.

Acknowledgements: this work was supported in part by grants CONACYT 154570, 50672 and PAPIIT-UNAM, IN220710-3. We also are grateful to Abigail Gonzalez Valdez from IIB/UNAM for the experimental support and finally to Javier Gallegos Infante (IFC/UNAM) for assistance in bibliographic materials.

Conference presentation: part of this paper was presented at the $3^{r d}$ International Conference on Acetic Acid Bacteria. Vinegar and Other products, 2012 Apr 17-20, Cordoba, Spain (http://www.uco.es/aab2012/).

Received for publication: 31 July 2012.

Revision received: 5 December 2012.

Accepted for publication: 10 December 2012.

This work is licensed under a Creative Commons Attribution 3.0 License (by-nc 3.0).

(C) Copyright S. Gómez-Manzo et al., 2013

Licensee PAGEPress, Italy

Acetic Acid Bacteria 2013; 2(s1):e2

doi:10.4081/aab.2013.s1.e2

\section{Introduction}

Fermentation industries producing vinegar, ascorbic acid, dihydroxyacetone, sorbose and other products of high commercial value, have exploited the tremendous metabolic power of acetic acid bacteria to oxidize a wide range of sugars, alcohols and aldehydes. ${ }^{1}$ Such oxidation reactions are termed oxidative fermentations, since they involve in the incomplete oxidation of substrates accompanied by accumulation of huge quantities of the oxidation products in the growth medium. ${ }^{2}$ In addition to the standard membrane respiratory complexes found in other aerobic bacteria, acetic acid bacteria possess a large and diverse set of membrane-bound dehydrogenases. These enzymes deliver electrons to the respiratory chain. ${ }^{2}$

Gluconacetobacter diazotrophicus is rather unique among the acetic acid bacteria because it carries out nitrogen fixation and is a true endophyte originally isolated from sugar cane..$^{3-6}$ Its presence in soils as free living bacteria has not been reported. In addition to its peculiar life style, $G$. diazotrophicus possesses a constitutive periplasmic oxidizing system for ethanol and acetaldehyde that is upregulated during N2-dependent growth. ${ }^{7}$ It is a Gram-negative bacterium and belongs to the acetic acid bacteria which consist of ten genera: Acetobacteer, Gluconobacter, Acidomonas, Gluconacetobacter, Asaia, Kozakia, Swaminathania, Saccharibacter, Neoasaia and Granulibacter of the Acetobactereaceae family. ${ }^{8}$ A set of dehydrogenases are overexpressed when $G$. diazotrophicus grows under nitrogen-fixing conditions. Among these are the PQQ1-dependent enzymes alcohol dehydrogenase (ADH) and aldehyde dehydrogenase (ALDH) which are located in the cytoplasmic membrane. ${ }^{7}$ These enzymes are oriented toward the periplasmic space and transfer electrons to ubiquinone $\mathrm{Q10}{ }^{9}$

\section{Bacterial alcohol dehydrogenases}

The bacterial alcohol dehydrogenases can be classified into three types: Class I (ADHs-I) is similar to methanol dehydrogenase of methylotrophic bacteria, these enzymes usually have a soluble quinoprotein with a $\alpha_{2} \beta_{2}$ structure located in the periplasm as in the case of Pseudomonas aeruginosa..$^{10}$ The optimum $\mathrm{pH}$ of the enzyme is high, requires ammonia or alkylamines as activators, oxidizes a wide range of alcohol substrates, including secondary alcohols and uses specific $c$ type cytochrome as electron acceptor. ${ }^{10}$ Class II alcohol dehydrogenases (ADHs-II) are quinohaemproteins, present as soluble monomers in the periplasm, contain two prosthetic groups, one molecule of pyrroloquinoline quinone (PQQ) and a single haem $C$ as in those enzymes described in Comamonas testosteroni, Comamonas acidovorans, $P$. putida, P. butanovora and Ralstonia eutropha. ${ }^{11-13}$ The optimum $\mathrm{pH}$ of 
these enzymes is around 7.5 and there is no requirement for an amine activator; notably, the enzymatic activity can be assayed with ferricyanide. ADHs-II enzymes have a wide specificity for primary and secondary alcohols, although they are unable to oxidize methanol; they also oxidize aldehydes. Azurin is the physiological electron acceptor ${ }^{14}$ (Figure 1A). The class III alcohol dehydrogenases are membrane-bound quinohaemproteins described exclusively in acetic acid bacteria, in conjunction with the aldehyde dehydrogenase, they are responsible for the oxidation of ethanol to acetic acid in vinegar production. ${ }^{14}$ The alcohol dehydrogenase of acetic acid bacteria is localized on the periplasmic side of the cytoplasmic membrane acting as a primary dehydrogenase linked to the respiratory chain via ubiquinone as the physiological electron acceptor. ${ }^{9}$ This class of enzymes does not require ammonium as an activator and has an optimum $\mathrm{pH}$ range between 4 and 6. Primary alcohols (C2-C6) are good substrates, while methanol or secondary alcohols are not oxidized. Some activity is seen with formaldehyde and acetaldehyde. ${ }^{15}$ Thus, its substrate specificity is relatively restricted when compared with type I and II alcohol dehydrogenases. ADHs class III enzymes are composed of two ${ }^{16-18}$ or three different subunits. ${ }^{9,19-21}$ Subunit I (71-80 kDa) is a quinoprotein containing one single haem $C$. The subunit II (43-53 kDa) is a multihaem protein containing three haems $C .^{18}$ It some cases, an additional 8-16 kDa protein (S-III) with an unknown function has been reported ${ }^{21}$ (Figure 1B).

In the acetic acid bacteria has been frequently observed that the ethanol-oxidizing ability can be easily changed or sometimes lost during their cultivation, especially in a prolonged shaking culture of $A$. aceti, in which spontaneous mutants incapable of oxidizing ethanol merge at high frequencies. ${ }^{22,23}$ The same kind of mutation has been observed in Acetobacter pasteurianus, in which spontaneous mutants deficient in ethanol oxidation can be obtained during a prolongedshaking culture with ethanol. ${ }^{24}$ In Gluconobacter suboxydans genetic instability has not been detected, ${ }^{19}$ instead a dramatic drop in $\mathrm{ADH}$ activity are seen under some cultivation conditions, especially at low $\mathrm{pH}$ and/or with high aeration, the presence of $\mathrm{ADH}$ with a very low activity level was reported (ADHi) also detected in Acetobacter aceti. ${ }^{19}$ In addition, Flores-Encarnacion et al. ${ }^{7}$ found that $G$. diazotrophicus PAL5 growing under $\mathrm{N}_{2}$-fixing conditions, in well-aerated media, possesses a respiratory system in which the dehydrogenases activities for ethanol, acetaldehyde and glucose were several fold increased as compared to $\mathrm{N}_{2}$-non fixer cells. Gómez-Manzo et al. ${ }^{18,25}$ have already isolated and purified a highly active $\mathrm{ADH}(\mathrm{ADHa})$ from $\mathrm{N}_{2}$-grown $G$. diazotrophicus, using forced aeration and natural acidifying conditions during culture. In the present review we make a comparative study of the molecular and catalytic properties of the active and inactive forms of $\mathrm{ADH}$ purified from $G$. diazotrophicus which allowed us to conclude that differences in the redox state of enzymes as purified could explain the notorious differences seen in the activity power of the compared enzymes.

\section{Molecular properties of the active and inactive alcohol dehydrogenases complexes}

Under high aerations and low pH Matsushita et al. ${ }^{19}$ purified an inactive ADH from Gluconobacter suboxydans which was 10 fold less activity than its active counterpart ADHa. ${ }^{19}$ In the same line, FloresEncarnacion et al. ${ }^{7}$ reported that under $\mathrm{N}_{2}$ fixing cultures of $G$. diazotrophicus with forced aeration and growth-dependent acidification, the ADH was largely expressed in its active form. Indeed, during the last purification step, the enzyme ADHa eluted as the major cytochrome $c$ containing fraction from a molecular exclusion column. A second and comparatively small peak containing cytochrome $c$ eluted fully separated. This second peak was poorly active on ethanol and therefore named as inactive $\mathrm{ADH}$ (ADHi). However, in Gluconobacter suboxydans ${ }^{19}$ the inactive enzyme elutes from a ionic exchange column as the major peak fraction with the active enzyme forming a shoulder. It seems that $\mathrm{ADHa}$ is an oligomeric association of three heterodimers, and therefore, the inactive ADH complex would be constituted of a single heterodimer. ${ }^{26}$ According to the significant differences in their respective molecular sizes; indeed, size calibration of the column chromatography suggested that ADHa is almost threefold $(330 \mathrm{kDa})$ the size showed by ADHi (120 kDa); these dates were confirmed by the oligomeric difference determined by size exclusion chromatography. ${ }^{26}$ In line with these observations, Gomez-Manzo et al. ${ }^{26}$ reported the native polyacrylamide gel electrophoresis (PAGE) analysis (Figure 2) of the purified ADHi
A

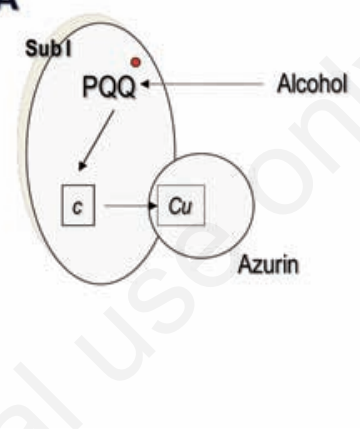

B

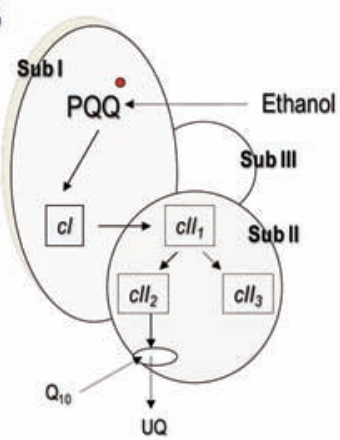

Figure 1. Hypothetical electron transfer in types II and III alcohol dehydrogenases (ADHs). (A) Estimated intramolecular and intermolecular electron transfer routes are shown in type II ADH (A) and type III ADH (B). In type III ADH, cI, $\mathrm{CII}_{1}, \mathrm{cII}_{2}$, and $\mathrm{cII}_{3}$ represent the 4 haem $c$ sites in subunit I and subunit II in ADH; Q10 may be present as bound quinone in vivo. In both cases the pyrroloquinoline quinone $(\mathrm{PQQ})$ is present in the semiquinone form $\left(\mathrm{PQQ}^{\circ}\right)$, and haems $\mathrm{c}$ are present in the reduced form (Adapted from Toyama et al. ${ }^{14}$ )

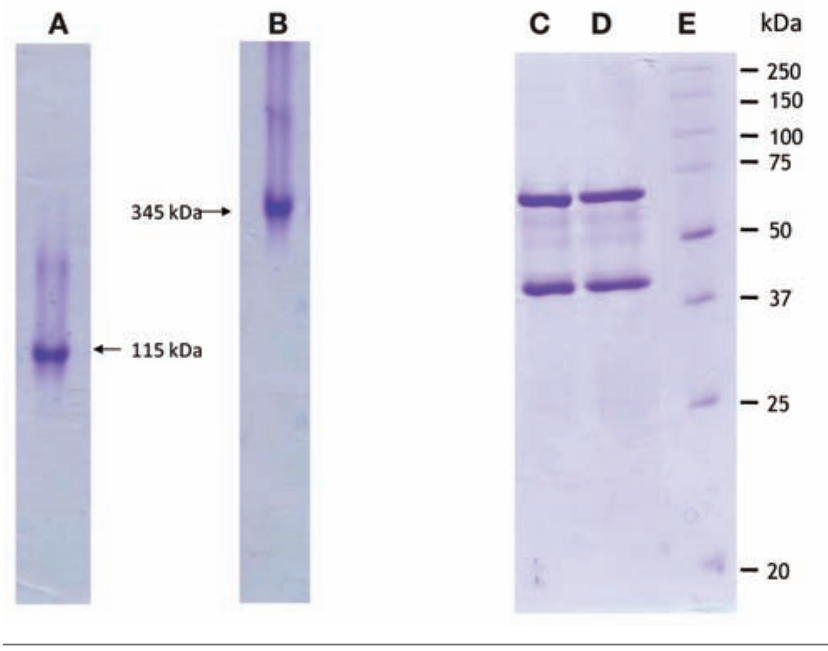

Figure 2. Polyacrylamide gel electrophoresis (PAGE) of the purified active (ADHa) and inactive alcohol dehydrogenase (ADHi) complexes. Native PAGE analyses of ADHi (A) and ADHa (B). SDS-PAGE analyses of the purified ADHi (C) and ADHa (D). Molecular weight standards (E) (Adapted from Gomez-Manzo et al. $\left.{ }^{26}\right)$. 
complex showed a homogeneous protein band with $\mathrm{Mr}=115 \mathrm{kDa}$ which turned out to be different at the previously obtained for the ADHa complex. In the same line, these authors compared both enzymes under denaturing conditions in SDS-PAGE, and found that the purified ADHi and ADHa were dissociated into two bands with relative molecular masses of $72 \mathrm{kDa}$ and $44 \mathrm{kDa}$ for SI and SII, respectively (Figure 2). It seems, that the active and inactive ADH-dimers of $G$. diazotrophicus consist of the same quaternary structure that in agreement with the similar oligomeric structure reported for the active and inactive ADHtrimers of Gluconobacter suboxydans..$^{27}$ In the same order, it's interesting to mention that both the ADHa and $\mathrm{ADHi}$ complex of $G$. diazotrophicus belongs to the group of membrane-ADHs that have two subunits. Interestingly, all the membrane-bound $\mathrm{ADHs}$ so far purified from Gluconacetobacter species ${ }^{9,16-21,28}$ are hetorodimers formed by 71-72 $\mathrm{kDa}$ and 43-45 $\mathrm{kDa}$ subunits (SI and SII, respectively). In contrast, among the few cases so far reported of Acetobacter and Gluconobacter species besides the catalytic core SI-SII, and additional 8-16 kDa subunit (SIII) has been described. Thus, the SI-SII heterodimeric structure found repetitively in Gluconacetobacter species could be a distinctive feature of the genus; in fact the SI-SII heterodimeric structure seems to be the minimal catalytic unit of membrane-bound ADHs of acetic acid bacteria.

\section{Spectroscopic characterization}

\section{Cytochromes}

Visible light spectroscopy of the purified ADHs enzymes showed absorption maxima at 552, 523, and $417 \mathrm{~nm}$ that could be assigned to the characteristic $\alpha, \beta$, and $\gamma$ absorption bands, respectively, of the reduced $c$-type cytochromes. Similar results have been reported in others ADHs, ${ }^{17-20,25,26,29}$ where they proposed that these are due to contaminating trace amounts of alcohol in the chemical used during the purification. In the same line, Gomez-Manzo et al. ${ }^{18}$ reported that it is possible that Triton X-100, having an ethoxyethanol residue, could act as a poor substrate maintaining the enzyme reduced along purification. Respect at the propose of this in a comparative study, the UV-VIS spectra of ADHa and ADHi (as prepared) purified from G. diazotrophicus (Figure 3A) showed significant differences in the endogenous reduction levels of the cytochromes $c$. While the cytochromes appeared fully reduced in ADHa (Figure 3A, trace a), reduction levels in $\mathrm{ADHi}$ were low (Figure $3 \mathrm{~A}$, trace b). In this sense, the UV-VIS spectra showed other variance in both enzymes. The ADHi complex showed a more intense signal at $350 \mathrm{~nm}$ than at $317 \mathrm{~nm}$ (Figure $3 \mathrm{~A}$ ). The spectral maximum at $350 \mathrm{~nm}$ correspond at the spectrum shown by the PQQ standard $^{30}$ and quinoproteins previously purified. ${ }^{31,32}$ This feature might account for the observed difference in the oxidation states of $\mathrm{PQQ}$ in both enzymes.

\section{Prosthetic group pyrroloquinoline quinone}

PQQ has been found in many different species of enzymes working on the dehydrogenation of the primary or secondary alcohols or sugars. ${ }^{15}$ The PQQ is non-covalently bound to the apoenzyme and these are called quinoproteins. The presence of $\mathrm{PQQ}$ in the $\mathrm{ADHs}$ from acetic acid bacteria has been confirmed by fluorescence spectroscopy, ${ }^{18}$ electron paramagnetic resonance (EPR), ${ }^{25}$ as well as by high-performance liquid chromatograpy (HPLC) analysis. ${ }^{26}$ Matsushita et al. ${ }^{19}$ observed by fluorescence spectra the presence of the $\mathrm{PQQ}$ in the native state with both ADHs. From this fluorescent study, they concluded that there is no difference in $\mathrm{PQQ}$-binding mode between active and inactive ADHs. Furthermore, Gomez-Manzo et al. ${ }^{18}$ purified the ADHa from $G$. diazotrophicus and confirmed the presence of the prosthetic group
PQQ by fluorescence spectroscopy, and corroborated by EPR. ${ }^{26}$ The EPR spectrum of ADHa showed a narrow signal centered at $g_{\text {iso }}=2.0034$, assigned to the $\mathrm{PQQ}$ semiquinone ${ }^{26}$ (Figure $3 \mathrm{~B}$ ). In line with these observations, the ADHi purified and characterized from $G$. diazotroph$i c u s^{26}$ demonstrated that the intensity of the signal showed by ADHi (as purified) in EPR was rather low as compared to that obtained for the ADHa complex of the same bacteria; ${ }^{25}$ however, after addition of dithionite to sample and recording the EPR spectrum of $\mathrm{ADHi}$, a more intense signal was obtained. ${ }^{26}$ This suggested that the $\mathrm{PQQ}$ prosthetic group in ADHi is mainly in its oxidized state, which is in contrast to the ADHa complex where $\mathrm{PQQ}$ was detected in its semiquinone form. EPR signals, with similar $g$-values and line widths, had been reported earlier for the PQQ-dependent enzymes from Pseudomonas aeruginosa ${ }^{33}$ and Comamonas testosteroni. ${ }^{13}$ Moreover, quantitative analysis performed by HPLC ${ }^{26}$ and confirmed the presence of one PQQ $(0.94 \pm 0.25)$ and one calcium ion $(1.10 \pm 0.05)$ per ADH heterodimer, which was detected by atomic absorption spectroscopy. This calcium ion has been widely reported that is required for cofactor binding and stabilization of the PQQ semiquinone radical. ${ }^{33-35}$ Additionally, the amino acid sequence of the ADHa from $G$. diazotrophicus indicates the presence of a specific binding site for the PQQ moiety in SU-I. ${ }^{14,36-38}$

The only, Gomez-Manzo and co-workers ${ }^{26}$ elucidated by HPLC analysis the redox state of the $\mathrm{PQQ}$ prosthetic group in ADHa and ADHi. For this purpose, $\mathrm{PQQ}$ were extracted from both enzymes by a methanolethanol mixture. For ADHa a single peak with a retention time of 4.5 min was obtained; noteworthy, the PQQ extracted from ADHi showed a single peak with a retention time of 6.8 min (Figure $3 \mathrm{C}$ ). On the other hand, they used a commercial PQQ which showed a retention time of $4.1 \mathrm{~min}$ and that shifted to $6.8 \mathrm{~min}$ after oxidation with $\mathrm{NH}_{4}{ }^{+}$peroxydisulfate (Figure 3C). With this results, these authors concluded that the PQQ in ADHi was present in its oxidized state (retention time 6.8 min) in contrast to ADHa where the $\mathrm{PQQ}$ was found in the semiquinone form (retention time $4.5 \mathrm{~min}$ ). ${ }^{12}$ This was the first report that showed a significant difference in the oxidation state of the PQQ prosthetic group in the catalytic sites of the active and inactive ADHs, respectively. It is tempting to speculate that this difference in the reduction state of $\mathrm{PQQ}$ might cause conformational differences that are instrumental to the catalytic process.

\section{[2Fe-2S] cluster}

In addition to the four c-type cytochromes present in the $\mathrm{ADHs}$ from acetic acid bacteria, an EPR signal at low temperature spectroscopy led to identified an iron-sulfur cluster associated with the membrane bound enzyme; which exhibited a rhombic signal with $g_{x y z}$ values at 2.007, 1.941, and 1.920 ( $g_{a v}$ 1.956). Comparable $g_{a v}$ values had been reported for the [2Fe-2S] cluster of benzene dioxygenase of Pseudomonas putida, aldehyde oxidoreductase of Desulfovibrio alaskensis, and the FhuF protein of E. coli..$^{39-41}$ The presence of a second type of iron center in ADH from G. diazotrophicus was supported by the quantitative determination of iron and acid-labile sulfur. ${ }^{18}$ The value of six $(5.90 \pm 0.15) \mathrm{Fe}$ and two acid-labile sulfur atoms $(2.06 \pm 0.10)$ per ADHa heterodimer protein are in agreement with the presence of four $c$-type cytochrome centers and one [2Fe-2S] cluster. In addition to, these authors determined the acid-labile sulfurs by the method of Beinert ${ }^{42}$ and found the presence of $2.02 \pm 0.1$ sulfur atoms per ADHi heterodimer. ${ }^{25}$ However, we report that the EPR spectrum of the purified ADHi showed no signal corresponding to the iron-sulfur cluster, it seems that the [2Fe-2S] cluster in ADHi must be in the oxidized form, which is a diamagnetic species.

Usually, ferredoxin-type [2Fe-2S] clusters are bound by cysteine residues to the protein. ${ }^{43}$ In this sense, the amino acid sequence of the membrane-bound ADH from $G$. diazotrophicus carries 11 cysteine 
residues, five of them in SU I and six in SU II. In SU II, all six cysteine residues are located in three $\mathrm{CXXCH}$ motifs required for covalent attachment of the three $c$-type cytochromes. ${ }^{14,36,44}$ In SU I, two of the cysteine residues are used for the classical $\mathrm{CXXCH}$ motif. This leaves three cysteine residues as potential ligands for the [2Fe-2S] cluster detected by EPR spectroscopy. Two of them are part of a sequence CCDxVNRG, conserved in both type II and type III quinohemoprotein alcohol dehydrogenases, including the type III ADH of $G$. diazotrophicus, which could serve as ligands for the [2Fe-2S] cluster, as reported for the [2Fe-2S] cluster of the FhuF protein in E. coli, ${ }^{40-41}$ or the [4Fe$4 \mathrm{~S}$ ] cluster in the assimilatory adenosine-50-phosphosulfate reductase of $P$. aeruginosa.$^{45}$ mentioned above, frequently ferredoxin-type [ $2 \mathrm{Fe}$ $2 \mathrm{~S}$ ] clusters are bound by four cysteine residues to the protein. ${ }^{43}$ As there are only three cysteine residues, in addition to the two used for attachment of one $c$-type cytochrome in SU I, Gomez-Manzo et al. ${ }^{25}$ assume that the fourth ligand of the [2Fe-2S] cluster must come from another amino acid residue, such as histidine or serine. ${ }^{25}$ This assumption is supported by the results published recently for the outer mitochondrial membrane protein mitoNEET. ${ }^{46-49}$ In this protein, the [2Fe-2S] cluster is coordinated by three cysteines and one histidine.

\section{Midpoint potentials of the prosthetic group of active and inactive alcohol dehydrogenases complex}

The determination of the redox potential of membrane bound enzymes is problematic. However, there are several studies where potentiometric titrations of hemes $c$ in ADHs complex from acetic acid bacteria were developed. ${ }^{20,18,26,28,50,51}$ Previous studies reporting on redox titration values of cytochromes $c(\mathrm{pH} 7.0)$ in purified $\mathrm{ADHs}$ are controversial. In Gluconobacter suboxydans, Ameyama and Adachi ${ }^{50}$ detected three cytochrome $c$ centers when the haem content was calculated from a pyridine spectrum; however, redox titration of the enzyme detected only two haem $c$ centers $\left(\mathrm{E}_{\mathrm{m}}=+260\right.$ and $\left.+340 \mathrm{mV}\right)$. Later on, Torimura et al. ${ }^{51}$ detected four cytochrome $c$ centers in the purified ADH of Gluconobacter suboxydans $\left(\mathrm{E}_{\mathrm{m}}=+101,+216,+370\right.$ and +401 $\mathrm{mV})$. While in Acetobacter methanolicus, Frébortova et al. ${ }^{20}$ detected four cytochrome $c$ centers $\left(\mathrm{E}_{\mathrm{m}}=-130,+49,+188\right.$ and $\left.+188 \mathrm{mV}\right)$. Later, Gomez-Manzo et al. ${ }^{18}$ detected four haem $c$ centers $\left(E_{m}=-64,-8,+185\right.$ and $+210 \mathrm{mV}$ ) in the purified ADH complex of $G$. diazotrophicus. More recently, Chavez-Pacheco et al. ${ }^{28}$ purified ah the $\mathrm{ADH}$ from $G$. xilynum and reported four haem $c$ centers $\left(E_{m}=-34,-6,+180\right.$ and $+344 \mathrm{mV}$, respectively). Thus, a comparison between the different cases is difficult. However, when compared the difference obtained in the potentiometric titrations between the ADHa and ADHi purified from $G$. diazotrophicus, the values detected in the ADHi were significantly more positive at the values obtained previously forts active counterpart ${ }^{26}$ (Figure 4).

Respect at the redox titration of the PQQ; earlier, Torimura and coworker ${ }^{51}$ had been obtained a value of $E_{m}=-167 \mathrm{mV}$ for the $\mathrm{PQQ} / \mathrm{PQQH}_{2}$ redox couple of the membrane-bound $\mathrm{ADH}$ of Gluconobacter suboxydans. In this sense, Duine and co-workers ${ }^{52}$ had reported $E_{m}=-218 \mathrm{mV}$ for the $\mathrm{PQQ} / \mathrm{PQQ}_{\mathrm{sq}}$ couple and $E_{m}=-242 \mathrm{mV}$ for the $\mathrm{PQQ}_{s q} / \mathrm{PQQH}_{2}$ couple in methanol dehydrogenase of Hyphomicrobium X. Later, Gomez-Manzo et $a l .{ }^{18}$ estimated a value of $E_{\mathrm{mPQ}}=-210( \pm 5) \mathrm{mV}$ (vs SHE) for the $\mathrm{PQQ} / \mathrm{PQQH}_{2}$ coupled by EPR spectroscopy in the membrane-bound $\mathrm{ADH}$ of G. diazotrophicus. Furthermore, recently Gomez-Manzo et al. ${ }^{18}$ reported the EPR-monitored oxidation-reduction titration of the [2Fe-2S] cluster in ADHa of $G$. diazotrophicus, where they found a value of $\mathrm{E}_{\mathrm{mFes}}=-250 \mathrm{mV}$.
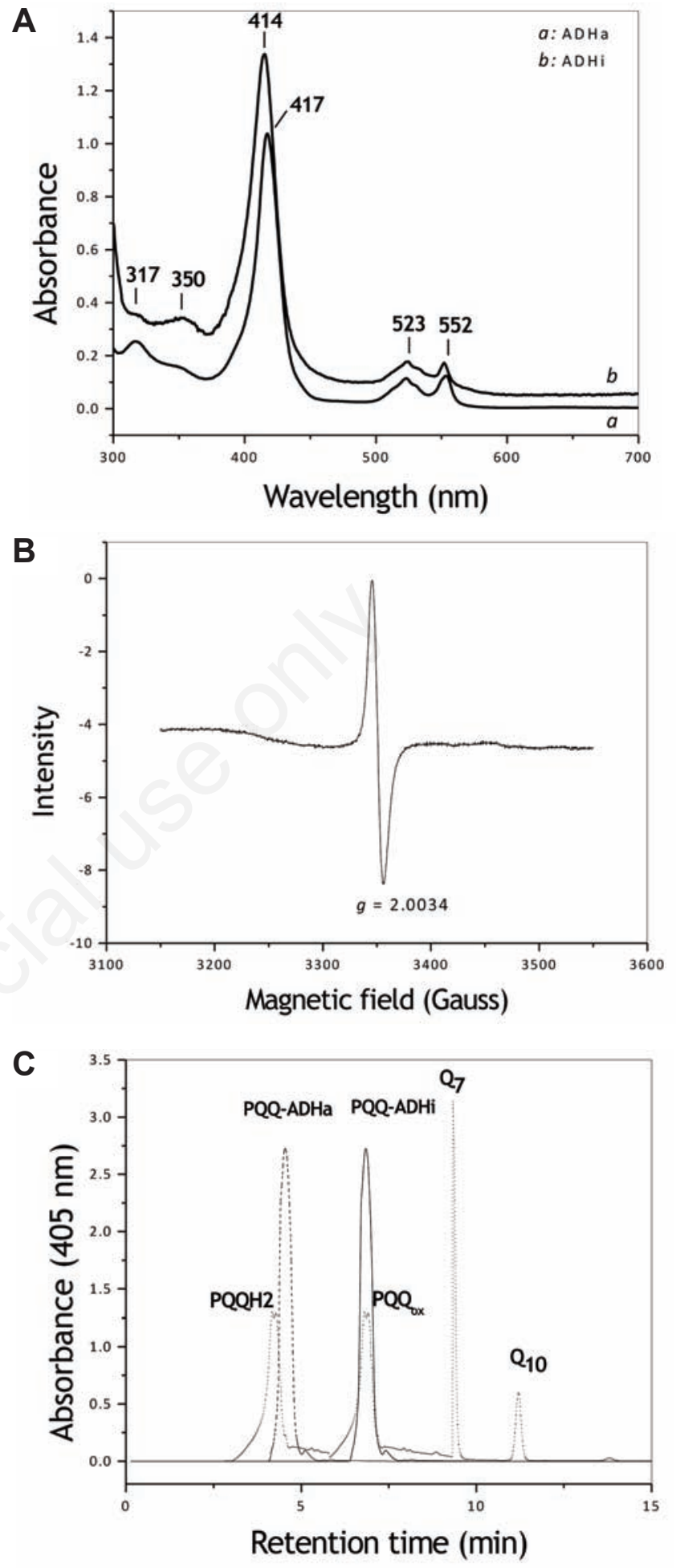

Figure 3. Redox differences in inactive (ADHi) and active alcohol dehydrogenase (ADHa) complexes. (A) Absorption UV-Vis spectra of the purified ADHa (trace a) and ADHi (trace b) complexes of G. diazotrophicus. (B) X-band electron paramagnetic resonance spectrum of the pyrroloquinoline quinone (PQQ) semiquinone radical in $\mathrm{ADH}$ of $G$. diazotrophicus. (C) Redox differences of the prosthetic group PQQ in ADHi and ADHa complexes. Reverse-phase HPLC of methanol-extracted PQQ associated to the purified $\mathrm{ADHa}$ and $\mathrm{ADHi}$ complexes of $G$. diazotrophicus. System calibration with the following commercial standards: PQQ in its commercial presentation (PQQH2) and after oxidation with ammonium peroxydisulfate (PQQ), Q7 and Q10 (retention times: 4.1, 6.8, 9.28 and $11.19 \mathrm{~min}$, respectively) (Adapted from Gomez-Manzo et al. ${ }^{18,26}$ ). 


\section{Intramolecular electron transfer in the inactive and active alcohol dehydrogenase complexes}

It is well known that the membranous alcohol dehydrogenases of acetic acid bacteria remove electrons from the substrate using its $\mathrm{PQQ}$ group as the first acceptor. ${ }^{53}$ The presence of a new cofactor: [2Fe-2S] in the S-I of ADHa of $G$. diazotrophicus, this cofactor is a key member for the intramolecular electron transfer sequence ${ }^{25}$ that according to its midpoint potential value it seems, that is acting as electron bridge between PQQ and the intramolecular cytochromes $c$ sequence of the ADH complex. Notably, the four cytochrome $c$ centers are redoxdependent chromogenic groups amenable for assessment of electron transfer kinetics within the ADH complex. Accordingly, the rate of intramolecular electron transfer evoked by ethanol was measured in both enzymes under the same experimental conditions. Comparable enzyme samples were first ferricyanide-titrated to the oxidized state. Then, ethanol was added and reduction of cytochromes $c$ was recorded as showed in the Figure 5A. These authors found that ethanol caused full reduction of the cytochrome $c$ centers in $\mathrm{ADHa}$, whereas in $\mathrm{ADHi}$ only one quarter of the total cytochrome $c$ content was reduced.

To assess the number of the cytochromes $c$ able to participate in the intramolecular electron transfer in the ADHi complex, Gomez-Manzo et $a l .^{26}$ titrated to its full reduced state with a dithionite-solution and then, successively the enzyme was oxidized with the hydrosoluble quinone-2 (Q2). As observed, close to $90 \%$ of the ferrocytochrome $c$ content of the enzyme was oxidized as revealed by the major decrease of wavelength signals at 419, 519 and $550 \mathrm{~nm}$. They proposed that although the catalysis of the ADHi fraction is severely limited, the four cytochromes $c$ centers in the intramolecular electron transfer sequence seems to remain active, delivering electrons to the $\mathrm{Q}_{2}$ electron acceptor (Figure 5B).

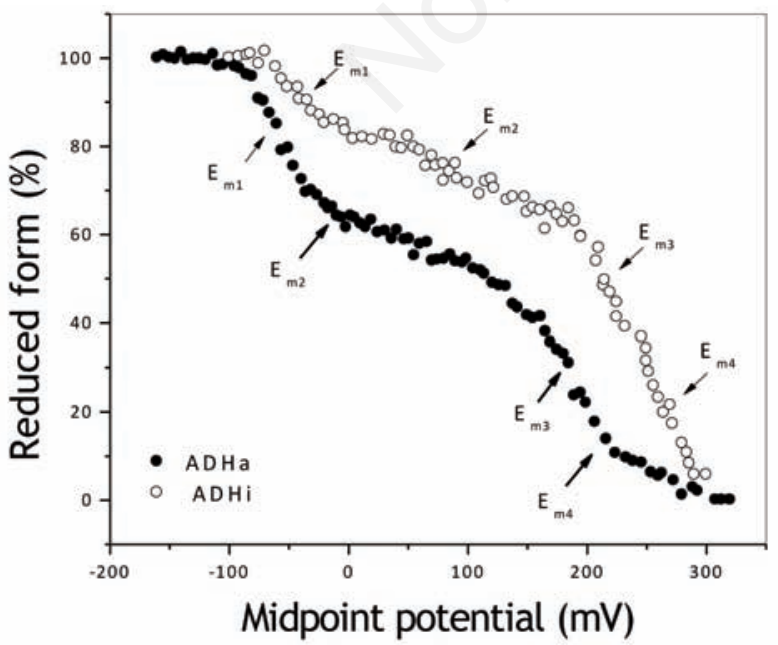

Figure 4. Spectroelectrochemical titration of c-type cytochromes in inactive $(\mathrm{ADHi})$ and active alcohol dehydrogenase $(\mathrm{ADHa})$ of G. diazotrophicus (Adapted from Gomez-Manzo et al. ${ }^{18,26}$ ).

\section{Conclusions}

In conclusion, the occurrence of inactive $\mathrm{ADH}$ seems to be strange with respect to alcohol oxidation, however Matsushita et al. ${ }^{19}$ have detected and characterized a second type of ADH (named inactive $A D H$ ) in Gluconobacter suboxydans and it can be generated by acidic (low-pH) or highly aerobic growth conditions, while active ADH can be predominant at neutral-pH or under low-aeration growth conditions. Respect to $G$. diazotrophicus has been observed that when the culture is obtained at acid $\mathrm{pH}$ and a high aeration, the major product was the active $\mathrm{ADH}$ and a very small amount of the inactive $\mathrm{ADH}$ was present. Also, has been observed that the regulation of the activity in the active and inactive $\mathrm{ADH}$ is given by the $\mathrm{pH}$ of culture medium; in the sense, it
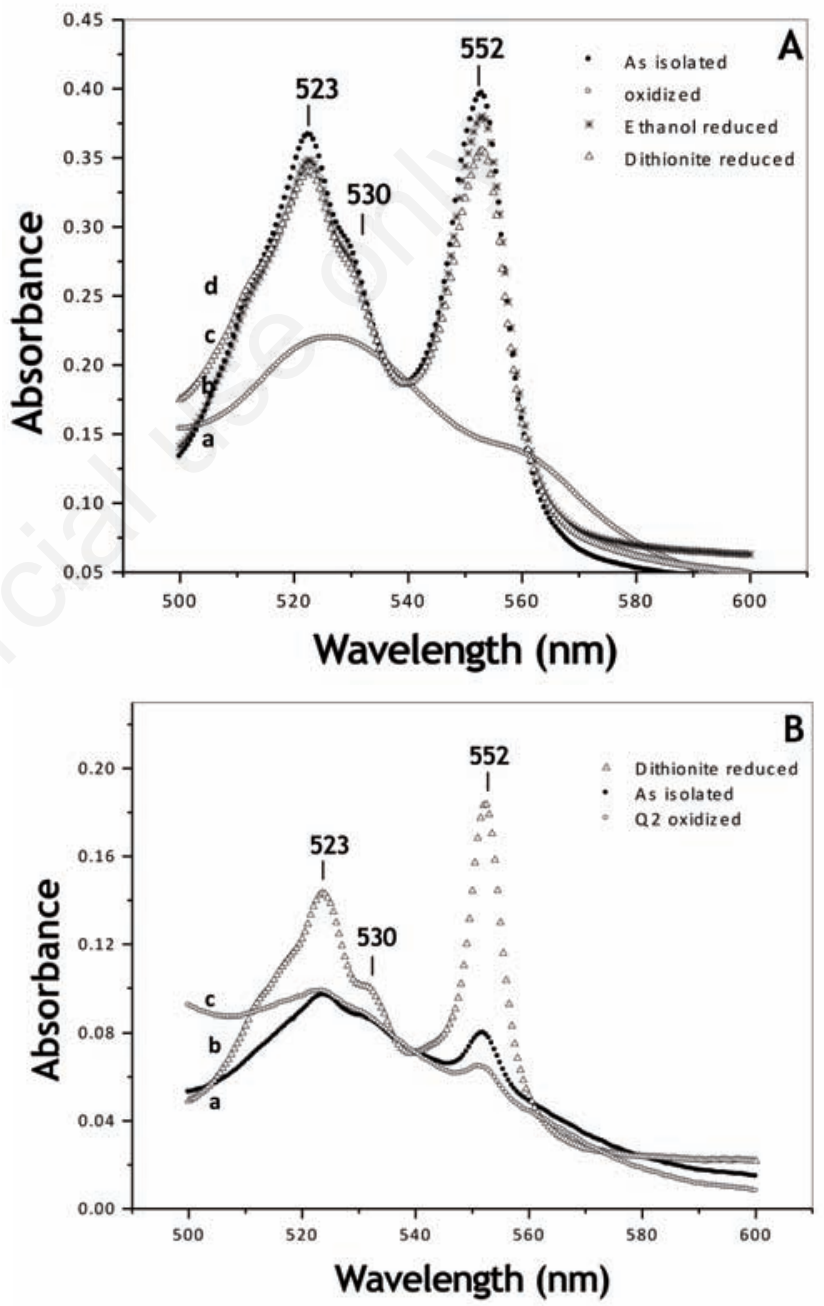

Figure 5. Redox properties of the purified active (ADHa) and inactive alcohol dehydrogenase (ADHi) complexes of $G$. diazotrophicus. (A) $(\mathrm{a}, \bullet)$ Spectrum of the ADHa complex as prepared. $(b, \circ)$ Spectrum of oxidized ADHa complex, obtained after titration of the enzyme with small amounts of potassium ferricyanide. $(\mathbf{c}, *)$ Spectrum of the ethanol-reduced enzyme by ethanol previously oxidized by ferricyanide. (d, $\Delta)$ Spectrum of the fully reduced enzyme obtained after addition of sodium dithionite to the enzyme previously reduced by ethanol. (B) (a, •) Spectrum of the ADHi complex as prepared $(b, \Delta)$ Spectrum of the enzyme after controlled-reduction with a dithionite solution (c, $\bigcirc)$ Spectrum of the oxidized enzyme obtained by addition of controlled amounts of soluble Q2 to the reduced enzyme obtained in (b). 


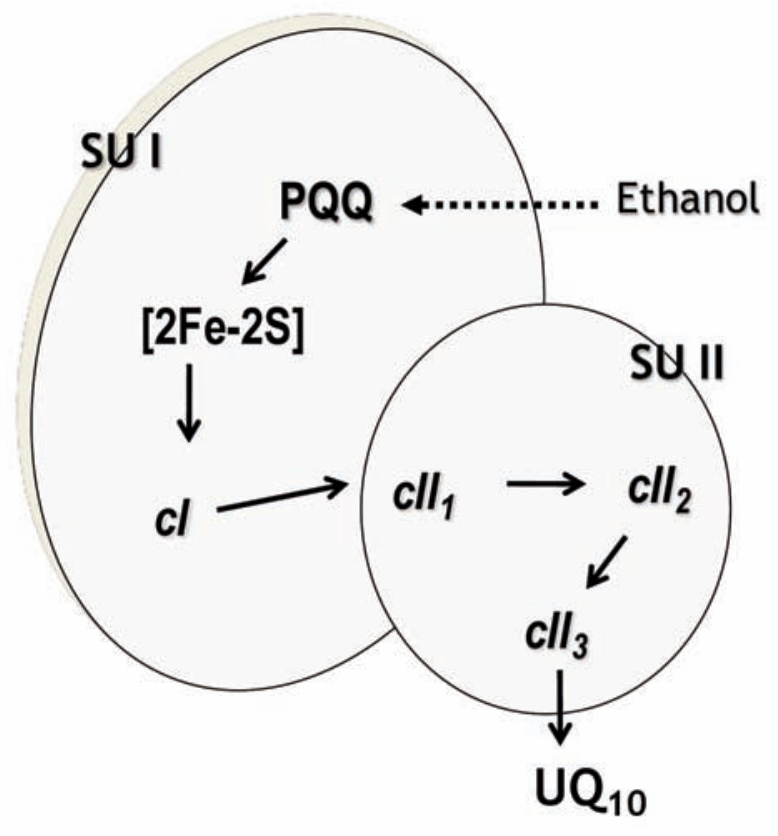

Figure 6. Hypothetical intra- and intermolecular electron transfer pathways in the heterodimeric membrane-bound alcohol dehydrogenase of $\boldsymbol{G}$. diazotrophicus. pyrroloquinoline quinone (PQQ), [2Fe-2S], and cytochrome cI are assigned to SU-I and cytochromes cII1, cII2, and cII3 to SU-II, interacting with UQ10.

has been determined that the optimum $\mathrm{pH}$ of the active $\mathrm{ADH}^{18}(\mathrm{pH}, 6.0)$ and also determined the optimum $\mathrm{pH}$ of the inactive $\mathrm{ADH}$ which was 4.0. These results suggest that at the beginning of the growth the ADH has the ability to oxidized the alcohols present in the medium; and that at the end of the phase of the growth (acid pH) the inactive $\mathrm{ADH}(15 \%$ of activity respect to the active $\mathrm{ADH}$ ) has the ability the oxidized the small quantity of alcohol remaining in the culture medium. ${ }^{26}$ Therefore, the results reported by Gomez-Manzo et al. ${ }^{26}$ suggest that even though the inactive ADH is isolated in its oxidized form, the four cytochromes $c$ are active their oligomeric composition and participate in intramolecular electron transfer from the PQQ to the endogenous ubiquinone. Therefore, they considered that the inactivity is mainly due to the difference in the oxidation state of the PQQ and not to a damage in any cytochrome $c$ as was proposed for the inactive ADH from the Gluconobacter suboxidans by Matsushita et al. ${ }^{19}$ The membranebound $\mathrm{ADHa}$ and $\mathrm{ADHi}$ from $G$. diazotrophicus carries three different types of redox-active centers, the $\mathrm{PQQ}$ cofactor, four $c$-type cytochromes, and one [2Fe-2S] cluster, which provide efficient intraand intermolecular electron transfer pathways needed for an efficient catalyst (Figure 6).

\section{References}

1. Adachi 0, Moonmangmee D, Shinagawa E, et al. New quinoproteins in oxidative fermentation. Biochim Biophys Act 2003;1647:10-7.

2. Matsushita K, Toyama H, Adachi O. Respiratory chains and bioenergetics of acetic bacteria. Adv Microb Physiol 1994;36:247-97.

3. Alvarez B, Martínez-Drets G. Metabolic characterization of Acetobacter diazotrophicus. Can J Microbiol 1995;41:918-24.
4. Cavalcante VA, Döbereiner J. A new acid-tolerant nitrogen fixing bacterium associated with sugarcane. Plant and Soil 1988;108:2331.

5. Gillis M, Kersters K, Hoste B, et al. Acetobacter diazotrophicus sp. nov., a nitrogen-fixing acetic bacterium associated with sugarcane. Inter J System Bact 1989;39:361-4.

6. Jiménez-Salgado T, Fuentes-Ramírez LE, Tapia-Hernández MA, et al. Coffea arabica L., a new host plant for Acetobacter diazotrophicus and isolation of other nitrogen fixing acetobacteria. Appl and Environ Microbiol 1997;63:3676-83.

7. Flores-Encarnación M, Contreras-Zentella M, Soto-Urzúa L, et al. The respiratory system and diazotrophic activity of Acetobacter diazotrophicus PAL5. J Bacteriol 1999;181:22:6987-95.

8. Yuzo Yamada, Pattaraporn Yukphan. Genera and species in acetic acid bacteria. Int J Food Microbiol 2008;125:15-24.

9. Matsushita K, Takaki Y, Shinagawa E, et al. Ethanol oxidase respiratory chain of acetic acid bacteria. Reactivity with ubiquinone of pyrroloquinoline quinone-dependent alcohol dehydrogenase purified from Acetobacter aceti and Gluconobacter suboxydans. Biosci Biotech Biochem 1992;56:304-10.

10. Schrover JM, Frank J, van Wielink JE, Duine JA. Quaternary structure of quinoprotein ethanol dehydrogenase from Pseudomonas aeruginosa and its reoxidation with a novel cytochrome $c$ from this organism. Biochem J 1993;290:123-7.

11. Jongejan A, Jongejan JA, Duine JA. Homology model of the quinohaemoprotein alcohol dehydrogenase from Comamonas testosteroni. Protein Eng 1998;11:185-98.

12. Groen BW, van Kleef MAG, Duine JA. Quinohaemoprotein alcohol dehydrogenase apoenzyme from Pseudomonas testosteroni. Biochem J 1986;234:611-5.

13. de Jong GA, Geerlof A, Stoorvogel J, et al. Quinohaemoprotein ethanol dehydrogenase from Comamonas testosteroni. Purification, characterization, and reconstitution of the apoenzyme with pyrroloquinoline quinine analogues. Eur J Biochem 1995;230:899905 .

14. Toyama H, Mathews FS, Adachi 0, Matsushita K. Quinohemoprotein alcohol dehydrogenases: structures, function, and physiology. Arch Biochem Biophys 2004;428:10-21.

15. Goodwin PM, Anthony C. The biochemistry, physiology and genetics of PQQ and PQQ-containing enzymes. Adv Microbial Physiol 1998;40:1-80.

16. Trcek J, Toyama K, Czuba J, et al. Correlation between acetic acid resistance and characteristics of $\mathrm{PQQ}$ dependent $\mathrm{ADH}$ in acetic acid bacteria. Appl Microbiol Biotechnol 2006;70:366-73.

17. Tayama K, Fukaya M, Okumura H, et al. Purification and characterization of membrane-bound alcohol dehydrogenase from Acetobacter polyoxogenes. Appl Microbiol Biotechnol 1989;32:181-5.

18. Gómez-Manzo S, Contreras-Zentella M, González-Valdez A, et al. The PQQ-Alcohol Dehydrogenase of Gluconacetobacter diazotrophicus. Int J Food Microbiol 2008;125:71-8.

19. Matsushita K, Yakushi T, Takaki Y, et al. Generation mechanism and purification of an inactive form convertible in vivo to the active form of quinoprotein alcohol dehydrogenase in Gluconobacter suboxydans. J Bacteriol 1995;177:6552-9.

20. Frébortova J, Matsushita K, Yakushi T, et al. Quinoprotein alcohol dehydrogenase of acetic acid bacteria: Kinetic study on the enzyme purified from Acetobacter methanolicus. Biosci Biotech Biochem 1997;61:459-65.

21. Kondo K, Horinuochi S. Characterization of the genes encoding the three component membrane bound alcohol dehydrogenase from Gluconobacter suboxydans and their expression in Acetobacter pasteurianus. Appl Environ Microbiol 1997;63:1131-8.

22. Muraoka H, Watabe, Ogasawara N. Effect of oxygen deficiency on acid production and morphology of bacterial cells in submerged 
acetic fermentation by Acetobacter aceti. J Ferment Technol 1982;60:171-80.

23. Ohmori S, Uozumi T, Beppu T. Loss of acetic acid resistance and ethanol oxidizing ability in an Acetobacter strain. Agric Biol Chem 1982;46:381-9.

24. Takemura H, Horinouchi S, Beppu T. Novel insertion sequence IS1380 from Acetobacter pasteurianus is involved in loss of ethanoloxidizing ability. J Bacteriol 1991;173:7070-6.

25. Gómez-Manzo S, Solano-Peralta A, Saucedo-Vázquez JP, et al. The membrane-bound quinohemoprotein alcohol dehydrogenase from Gluconacetobacter diazotrophicus PAL5 carries a [2Fe-2S] cluster. Biochemis 2010;49:2409-15.

26. Gómez-Manzo S, González-Valdez AA, Oria-Hernández J, et al. The inactive and active forms of the PQQ-alcohol dehydrogenase of Gluconacetobacter diazotrophicus differ in the redox state of their respective prosthetic groups. FEMS Microbiol Lett 2012;328:106-13.

27. Matsushita K, Yakushi T, Toyama H, et al. Function of multiple heme $\mathrm{C}$ moieties in intramolecular electron transport and ubiquinone reduction in the quinohemoprotein alcohol dehydrogenase-cytochrome $c$ complex of Gluconobacter suboxydans. J Biol Chem 1996;271:4850-7.

28. Chavez-Pacheco JL, Contreras-Zentella M, Membrillo-Hernandez J, et al. The quinohaemoprotein alcohol dehydrogenase from Gluconacetobacter xylinus: molecular and catalytic properties. Arch Microbiol 2010;192:703-13.

29. Adachi 0, Miyagawa E, Shinagawa E, et al. Purification and characterization of particulate alcohol dehydrogenase from Acetobacter aceti. Agric Biol Chem 1978;42:2331-40.

30. Dekker RH, Duine JA, Frank J, et al. Covalent addition of H2O, enzyme substrates and activators to pyrrolo-quinoline quinone, the coenzyme of quinoproteins. Eur J Biochem 1982;125:69-73.

31. Anthony C, Zatman LJ. The microbial oxidation of methanol. Purification and properties of the alcohol dehydrogenase of Pseudomonas sp. M27. Biochem J 1967;104:960-9.

32. Groen BW, van Kleef MAG, Duine JA. Quinohaemoprotein alcohol dehydrogenase apoenzyme from Pseudomonas testosteroni. Biochem J 1986;234:611-5.

33. Kay CWM, Mennenga B, Gorisch H, Bittl R. Structure of the pyrroloquinoline quinone radical in quinoprotein ethanol dehydrogenase. J Biol Chem 2006;281:1470-6.

34. Duine JA, Jongejan JA. Quinoproteins, enzymes with pyrroloquinoline quinone as cofactor. Annu Rev Biochem 1989;58:403-26.

35. Sato A, Takagi K, Kano K, et al. Ca2+ stabilizes the semiquinone radical of pyrroloquinoline quinone. Biochem J 2001;357:893-8.

36. Bertalan M, Baldani IJ, Ferreira P, RioGene consortium. Gluconacetobacter diazotrophicus Pal5 complete genome.
Bethesda, MD: National Institute of Health; 2007.

37. Anthony $\mathrm{C}$. The structure of bacterial quinoprotein dehydrogenases. Int J Biochem 1992;24:29-39.

38. Anthony C. Pyrroloquinoline quinone (PQQ) and quinoprotein enzymes. Antiox Red Signal 2001;3:757-74.

39. Andrade SLA, Brondino CD, Feio J, et al. Aldehyde oxidoreductase activity in Desulfovibrio alaskensis NCIMB 13491 EPR assigment of the proximal [2Fe-2S] cluster to the Mo site. Eur J Biochem 2000; 267:2054-61.

40. Müller K, Matzanke BF, Schünemann V, et al. FhuF, an iron-regulated protein of Escherichia coli with a new type of [2Fe-2S] center. Eur J Biochem 1998;258:1001-8.

41. Matzanke BF, Anemüller S, Schünemann V, et al. FhuF, part of a siderophore-reductase system. Biochem 2004;43:1386-92.

42. Beinert H. Semi-micro methods for analysis of labile sulphide and of labile sulphide plus sulfane sulphur in unusually stable ironsulfur proteins. Anal Biochem 1983;131:373-8.

43. Meyer J. Iron-sulfur protein folds, iron-sulfur chemistry, and evolution. J Biol Inorg Chem 2008;13:157-70.

44. Darwin A, Hussain H, Griffiths L, et al. Regulation and sequence of the structural gene for cytochrome c552 from Escherichia coli, not a hexaheme but a $50 \mathrm{kDa}$ tetraheme nitrite reductase. Mol Microbiol 1993;9:1255-65.

45. Chartron J, Carroll KS, Shiau C, et al. Substrate recognition, protein dynamics, and iron-sulfur cluster in Pseudomonas aeruginosa adenosine-50-phosphosulfate reductase. J Mol Biol 2006;364:15269.

46. Paddock ML, Wiley SE, Axelrod HL, et al. MitoNEET is a uniquely folded $2 \mathrm{Fe}-2 \mathrm{~S}$ outer mitochondrial membrane protein stabilized by pioglitazone. Proc Natl Acad Sci U S A 2007;104:14342-7.

47. Wiley SE, Paddock ML, Abresch EC, et al. The outer mitochondrial membrane protein mitoNEET contains a novel redox-active $2 \mathrm{Fe}-2 \mathrm{~S}$ cluster. J Biol Chem 2007;282:23745-9.

48. Hou X, Liu R, Ross S, et al. Crystallographic studies of human mitoNEET. J Biol Chem 2007;282:33242-6.

49. Lin J, Zhou TZ, Ye K, Wang J. Crystal structure of human mitoNEET reveals distintic groups of iron-sulfur proteins. Proc Natl Acad Sci U S A 2007;104:14640-5.

50. Ameyama M, Adachi 0. Alcohol dehydrogenase from acetic acid bacteria, membrane-bound. Methods Enzymol 1982;89:450-7.

51. Torimura M, Kano K, Ikeda T, Ueda T. Spectroelectrochemical characterization of quinohemoprotein alcohol dehydrogenase from Gluconobacter suboxydans. Chem Lett 1997;525-6.

52. Duine JA, Jongejan JA. Quinoproteins, enzymes with pyrroloquinoline quinone as cofactor. Ann Rev Biochem 1989;58:403-26. 